\title{
EDITORIAL
}

\section{Healing by Skeptical Trainee Healers}

\author{
ALEX HANKEY, Ph.D. ${ }^{1}$ and SARAH MCCRUM, M.A. ${ }^{2}$
}

$\mathbf{H}$ ealing is a phenomenon that until quite recently scientists rejected out of hand. Now it seems that, despite grave doubts as to the possibility, even skeptical studentsincluding both scientists and nonscientists — can be trained to carry it out effectively, if only on mice (which are presumably easier to heal than humans). Bengtson's account (pp. 317-327) of six experiments on healing mice of adenocarcinomas, with very high success rates (average over $90 \%$ ) seem to imply that anyone anywhere can become a trained healer - at least of mice, if not of human beings.

For such a rate of full remission in experimental mice to be achieved by inexperienced 'healers' is remarkable, to say the least. Bengtson's results (pp. 317-327) are therefore of fundamental scientific importance. It is to be hoped that many attempts will be made to reproduce them at Master's and even undergraduate levels. They are apparently simple, and can be carried out in an appropriate time-frame. Also they have significant implications for many different fields of complementary and alternative medicine (CAM).

Healing is a phenomenon that is on the increase, with increasing numbers of people participating in it. It is subject to increasing amounts of active research ${ }^{1-4}$ and comment. ${ }^{5}$ Bengtson's paper shows that simple experiments on healing can shed light on core problems in understanding CAM, and indeed in biology and medicine: the utility of randomized control trials in the present day and age ${ }^{6}$; the occurrence of quantum effects in patient-practioner relations ${ }^{7,8}$ the phenomenon of placebo ${ }^{9}$ (N.B.: expectation of cure cannot be induced in mice, so no placebo applies to his experiments ${ }^{10}$ ); the nature of the life force or qi. ${ }^{11}$ These implications show that healing may contribute in key ways to the present rapid development of understanding of CAM. In particular, Bengtson's theory of resonant bonding can be linked with theories of life energy, common in many fields of complementary medicine, especially Traditional Chinese Medicine (TCM). There are many well-known techniques and treat- ments that stimulate life energy in ways appropriate to produce cure. ${ }^{4,11}$ All cures, spontaneous recovery and "remission," can then be attributed to changes in "life energy."

Biomedicine still does not recognize life energy, though well-developed theories are now available. ${ }^{12}$ Such cures are therefore referred to as "placebo-induced". In systems acknowledging the existence of life energy, every change in health is the result of that-placebo effect included. Even "healing" can be equated with an "induced placebo effect." There is really no difference.

Among Bengtson's more revealing conclusions are that the emotional state of the skeptical healer seems to affect outcome consistently. That healing occurs more easily when patient and/or practitioner are at ease is a well-established phenomenon. Much emphasis is placed on the value of being deeply relaxed since that facilitates the flow of qi energy. ${ }^{11}$ Another observation in his experiments (Control Problem 1) was that healing occurred spontaneously when trained healers merely saw groups of control animals in which some had already died. This can be attributed to a well-known phenomenon among healers-that once the training is there, healing can take place spontaneously even without their active intention. It is as if the healing energy flows from a "higher potential" to a "lower potential." Indeed, this supports Tiller's recent observations that human attention can somehow increase a measurable kind of "free energy" associated with the the life force or $q i{ }^{13,14}$

Like all radical, pioneering research, Bengtson's work raises many further questions - more than it answers. Many offer new avenues of research with potentially far-reaching implications. For example, if it is possible for inexperienced, skeptical healers using a simple technique learned over a few weeks to achieve $90 \%-100 \%$ healing in mouse carcinomas, what would be the influence of such healers on human cancers?

Preliminary work on this could extend the mouse research to investigate any difference in results between (1) mice in-

\footnotetext{
${ }^{1}$ Manipal Universal Learning, Bangalore, India.

${ }^{2}$ Phenomena Academy, Takaro, Te Anau, New Zealand.
} 
jected with disease and, (2), mice naturally contracting disease. If naturally occurring carcinomas in ordinary mice can be cured, that would be grounds for extending to humans. One suspects that the rate of success would be lower for humans, an observation that would then challenge theoretical explanation in terms of life force potential. ${ }^{13}$

Human research could be simpler and less restrictive: How effective could healing be for maintaining health, not just for treating carcinomas or mere disease? Apparently disparate results have recently been reported for Johrei healing, ${ }^{1-3}$ but the study of its effects on eczema ${ }^{3}$ only marginally failed to reach significance. Combined with the first two studies, ${ }^{1,2}$ this indicates a fair success rate. Bengtson's system would be worth evaluating for human subjects.

When understood in the context of life energy, the combination of healing and Bengtson's "resonant bonding" concept of collective health points to the possibility of a radically different systems of national health care. First, if any skeptical person can be trained to heal, anyone could become a "healer." Health maintenance would be in the hands of ordinary people. Were indicated success rates maintained, its effectiveness and reliability might make it the treatment of choice. Second, any system that could increase the strength of the quantum coherence (entanglement) in the general population ${ }^{14}$ would enable everyone to benefit from healing, and improve health generally.

We can envision that under such a universal system of healing, the health of the general population will constantly improve as a result of bonding and expanding resonance between ever increasing numbers of healthy members of the population, healing the sick. The strong will pull up the weak in a spiral of improving health, until health becomes natural and sickness belongs to history.

\section{REFERENCES}

1. Reece K, Schwartz GE, Brooks AJ, Nangle G. Positive well being changes associated with giving and receiving Johrei healing. J Altern Complement Med 2005;11:455-457.

2. Brooks AJ, Schwartz GE, Reece K, Nangle G. The effect of Johrei healing on substance abuse recovery. J Altern Complement Med 2006;12:625-631.
3. Canter PH, Brown LB, Greaves C, Ernst E. Johrei family healing: A pilot study. Evid Based Complement Alternat Med 2006;3:533-540.

4. Abbott NC. Healing as a therapy for human disease: a systematic review. J Altern Complement Med 2000;6:159-169.

5. Hankey A. CAM and the phenomenology of pain. Evid Based Complement Alternat Med (eCAM) 2006;3:139-141.

6. Milgrom L. Are random controlled trials (RCTs) redundant for testing the efficacy of homeopathy? A critique of RCT methodology based on homeopathy. J Altern Complement Med 2005; 11:831-838.

7. Walach H. Generalized entanglement: A new theoretical model for understanding complementary and alternative medicine. J Altern Complement Med 2005;11:549-559.

8. Milgrom LR. "Torque-like" action of remedies and diseases on the vital force, and their consequences for homeopathic treatment. J Altern Complement Med 2006;12:915-929.

9. Tiller WA. Human psychophysiology, macroscopic information entanglement and the placebo effect. J Altern Complement Med 2006;12:1015-1027.

10. Evans D. Placebo: The Belief Effect. London: HarperCollins, 2003.

11. Hankey A, McCrum S. Qigong: Life energy and a new science of life [letter]. J Altern Complement Med 2006;12: 841-842.

12. Hankey A. The life force. Hpathy.com Ezine Dec 2006. www. hpathy.com/research/hanky-life-force.asp. Accessed March 16, 2007.

13. Tiller WA, Dibble WE. Toward general experimentation and discovery in conditioned laboratory and complementary and alternative medicine spaces: Part V. J Altern Complement Med 2007;13:133-150.

14. Hankey A. The thermodynamics of healing, health, and love. J Altern Complement Med 2007;13:5-8.
Address correspondence to: Alex Hankey, Ph.D. Hethe House Cowden, Edenbridge Kent TN8 7DZ United Kingdom E-mail: alexhankey@googlemail.com 